\title{
cálculo no lineal de estructuras reticuladas y laminares
}

JESUS ORTIZ HERRERA, Dr. Ingeniero de Caminos Jefe del Departamento de Programación y Cálculo electrónico de MART-2 Gabinete técnico, S. A

sinopsis

\section{INTRODUCCION}

El objeto de la presente exposición es el de mostrar aquellos temas del cálculo no lineal de estructuras susceptibles de ser incorporados ya, de una forma eficiente y práctica, al análisis de estructuras cuya complejidad o importancia sugiera su estudio mediante métodos más perfeccionados y adecuados a la realidad que los del cálculo elástico y cálculo plástico tradicionales. Al igual que estos últimos (con mayor motivo, dado su mayor grado de sofisticación) su puesta en práctica obliga de hecho a la utilización del cálculo electrónico. Pero, contando con dicho medio de cálculo, el análisis no lineal de estructuras es ya un instrumento práctico, cuya utilización requiere únicamente (respecto del cálculo elástico) algunos datos más que fijen el comportamiento anelástico de las piezas; y un cierto incremento de los tiempos de uso del ordenador. El mostrar esto es precisamente la idea del presente trabajo, que incluye una selección de las numerosas pruebas efectuadas por el autor, mediante técnicas de cálculo propias.

La utilización del cálculo no lineal de estructuras no debe interpretarse simplemente como una mejora de los métodos de cálculo permitiendo una mayor adecuación al comportamiento real de las estructuras; es además una herramienta susceptible de ampliar el campo de acción del proyectista en su bús- queda de formas estructurales más constructivas y económicas, puesto que en muchos casos es posible obtener el grado necesario de seguridad frente al agotamiento de la estructura (sin situarse, dentro de ciertos márgenes, en malas condiciones respecto a los restantes estados límites), sin tener que ajustar el dimensionamiento de las piezas a distribuciones de esfuerzos de carácter «elástico». El cálculo no lineal permite efectuar un análisis riguroso de las diferentes fases de comportamiento anelástico de las estructuras, sin caer en las limitaciones del cálculo plástico tradicional.

La presente exposición no se limita al caso de estructuras compuestas de barras, ya que el cálculo no lineal es utilizado también para el análisis de estructuras superficiales, tal como veremos a continuación para el caso de las láminas de revolución de hormigón armado. Se entra así en un dominio reservado actualmente a los ensayos en modelo reducido, con ventaja respecto a éstos en costes y tiempos de ejecución; e incluso con ventaja también en lo relativo a la bondad de los resultados si la estructura real pone en juego su comportamiento anelástico, ya que la reproducción de éste por el modelo es de indudable dificultad y rara vez llevada a la práctica.

Por razones de claridad y extensión no se insiste en el aspecto matemático de las técnicas de cálculo, tema objeto de trabajos posteriores. 


\section{LIMITACIONES DEL CALCULO PLASTICO "TRADICIONAL"}

El procedimiento actualmente aceptado de dimensionar secciones y piezas en base a criterios de rotura, pero partiendo de esfuerzos determinados elásticamente (o, a lo sumo, con redistribuciones incorporadas de forma apriorística) viene impuesto por el estado actual de las técnicas de cálculo, pero es claro que presenta una grave contradicción interna en sus principios si la estructura no es isostática. De esa forma, el análisis en agotamiento puede presentar deficiencias; y cuando menos, se limita el campo de acción en el diseño de la estructura.

Los métodos "tradicionales" de cálculo en agotamiento los proporciona la Teoría de la Plasticidad, pero son bien conocidas sus limitaciones en cuanto la deformabilidad plástica del material no es muy acusada o las acciones son total o parcialmente fluctuantes. En ambos casos es preciso limitar de alguna forma las redistribuciones de esfuerzos respecto de las leyes elásticas, bien para no agotar la deformabilidad máxima real de las zonas plastificadas, o bien para evitar que, por efecto de las repeticiones de las cargas, las deformaciones plásticas residuales se acumulen de forma creciente (sin límite, y para repeticiones de carga en número relativamente reducido) (1). Otros efectos no tenidos en cuenta por la Teoría clásica de la plasticidad son:

- El carácter no localizado de las «rótulas plásticas" y la influencia de las deformaciones elásticas de las zonas no plastificadas. Incluso en estructuras con importante deformabilidad plástica, como son por ejemplo las constituidas por elementos mixtos (hormigón-acero estructural) normalmente proporcionados, si la longitud de las piezas es media o elevada, la deformabilidad elástica de las zonas comprendidas entre una rótula plástica teórica

(1) Un estudio sobre este fenómeno (inestabilidad de la deformación plástica) puede verse en las referencias 3 (Massonnet-Save) y 4 (Freudenthal-Geiringer). Como regla práctica para evitarlo, puede darse la de que no se produzcan plastificaciones bajo la actuación de las acciones características, cuando esfuerzos y secciones se calculen elastoplásticamente (bajo las cargas mayoradas]. Si el cálculo de esfuerzos es elástico, puede omitirse dicha comprobación; y también, normalmente, en el caso de estructuras poco importantes y con valores moderados de la relación sobrecarga/carga permanente. y otras dos adyacentes de signo contrario puede agotar la deformabilidad de estas últimas, sin que en la primera se haya provocado la rotación necesaria para que se desarrolle el momento de agotamiento de la misma; esto reduce obviamente el nivel de carga admitido por la estructura. En estructuras de hormigón este efecto es, por supuesto, más acusado.

- Salvo en vigas continuas y en pórticos con flexión predominante, la interacción entre axil y flector puede afectar de forma considerable a los valores últimos de los esfuerzos y de las deformaciones. Normalmente, en el cálculo plástico de estructuras lo que se hace es reducir el valor del momento plástico en función del axil; pero los límites de las rotaciones plásticas pueden asimismo reducirse, lo que afecta al completo desarrollo del mecanismo de colapso.

En rigor deberían considerarse plastificaciones simultáneas por axil y flector, y operar con los valores "reales" de las deformaciones plásticas, tanto giros como elongaciones. El esfuerzo cortante es también susceptible de interactuar con los valores últimos de los esfuerzos normales e, incluso, de producir deformaciones anelásticas de consideración (2).

- La influencia de posibles predeformaciones de una parte de los elementos, sobre los límites de los giros plásticos de las rótulas teóricas.

- La influencia de los fenómenos de segundo orden en deformaciones. En las piezas de poca importancia es aceptable el estudio separado del estado límite de inestabilidad (de acuerdo con las teorías del pandeo anelástico, si ello es necesario] y del estado límite de rotura. Pero, en rigor, la estructura debe analizarse con la consideración simultánea e interactiva de las deformaciones anelásticas bajo los esfuerzos que solicitan a sus secciones, y de los fenómenos de segundo orden, ya que estos últimos pueden modificar aquellos esfuerzos y, asimismo, el incremento anelástico de los movimientos agudiza lógicamente los fenómenos de segundo orden. Entonces el pandeo aparece como un caso particular en el que esfuerzos y movimientos no se estabilizan para un valor dado de las cargas; pero en otros casos la situación de agotamiento se alcanza sin pan-

(2) V. ref. 8 (J. Martínez Calzón). 
deo, aunque para cargas diferentes que las previstas dentro del cálculo de primer orden en deformaciones.

Si a estos defectos teóricos añadimos que el cálculo plástico de estructuras complejas es de un nivel de laboriosidad similar al propio cálculo elástico (1), se comprende que el interés práctico de dicho tipo de cálculo se restringe a un grupo limitado de estructuras (2), dada la actual tendencia a la combinación prolija de materiales y de formas.

\section{ANALISIS NO LINEAL DE ESTRUCTURAS DE BARRAS}

En contraposición al cálculo elástico y al plástico, se denomina genéricamente cálculo «no lineal" de estructuras al que tiene en cuenta los diferentes efectos citados en el apartado anterior, resumibles en: la influencia del comportamiento anelástico real de los materiales (diferente del comportamiento elástico y del ideal rígido-plástico) y la influencia de los cambios de geometría de la estructura (debidos a la deformación) en las condiciones de equilibrio y de compatibilidad de deformaciones. Normalmente se mantiene el carácter "determinístico" del cálculo; pero esto no es inconveniente alguno si la seguridad de la estructura se estudia con criterios semi-probabilísticos (que sólo afectan a los datos a introducir en un algoritmo determinístico); no obstante, en casos especiales puede ser necesario acudir a técnicas de simulación, a fin de efectuar un análisis probabilístico riguroso de la seguridad de la estructura.

Los métodos más extendidos actualmente para el cálculo no lineal de estructuras de

(1) Los métodos generales de cálculo plástico de estructuras de barras se basan en los algoritmos de la programación lineal [v. refs. 1 (Livesley) y 10 (Simonnard]; y no suponen una ventaja sustancial respecto a los métodos elásticos (siempre que se utilice el método de la matriz flexibilidad si la estructura es de un grado de hiperestaticidad pequeño) .

(2) Estructuras de acero estructural con amplio escalón de fluencia (y cierto tipo de estructuras mixtas), con cargas no dinámicas y relaciones sobrecarga/carga permanente moderadas, con esfuerzos predominantemente de flexión (reduciendo adecuadamente los momentos plásticos en función de axiles y cortantes) y respetando condiciones exigentes frente a la posible aparición de fenómenos de segundo orden. $Y$ con grado de hiperestaticidad pequeño, salvo utilización de ordenadores. barras pueden calificarse de artificiosos y de poco versátiles, tanto desde el punto de vista del comportamiento de los materiales como de las tipologías y geometrías de las estructuras consideradas. El autor del presente trabajo ha investigado en la línea de la generalización de los algoritmos "matriciales" del cálculo elástico (3), obteniendo un método altamente eficiente y general para la evaluación de la "respuesta" no lineal de cada barra prismática recta componente de la estructura; las ecuaciones generales de esta última se forman (a partir de las ecuaciones que fijan aquella respuesta particular de cada barra) mediante técnicas similares a las habituales en el cálculo matricial elástico (con ciertas modificaciones); con lo cual el algoritmo resultante es de alta rentabilidad conceptual y práctica, por su gran generalidad y relativamente sencilla programación.

Además de la definición geométrica y topológica de la estructura y de la de sus condiciones de sustentación, comunes ambas con el análisis elástico, el cálculo no lineal requiere como datos las leyes de deformación de las piezas en sus diferentes estados, lineales y anelásticos. Manteniendo la hipótesis de deformación plana, dichas leyes (leyes "constitutivas") pueden establecer directamente entre deformaciones globales de las secciones (elongación unitaria, $\varepsilon$, y curvatura, $\chi$, de la directriz) y esfuerzos (axil, $\mathbf{N}$, y flector, $\mathbf{M}$ ). Si las deformaciones por cortante son de consideración, su efecto puede tenerse hasta cierto punto en cuenta sin más que efectuar una corrección adecuada de las leyes constitutivas que se deducen de la hipótesis de deformación plana (4); pero cuando las dimensiones transversales de las piezas dejan de ser pequeñas en relación a las longitudinales, ya no es posible seguir manteniendo leyes esfuerzos-deformaciones globales, y el análisis debe efectuarse con criterios localizados: es decir, hay que abandonar la teoría de las estructuras de barras prismáticas y acudir a la Mecánica de los medios continuos.

La estimación de las leyes esfuerzos-deformaciones es sencilla y rápida con la ayuda del cálculo electrónico; existen gran cantidad de trabajos sobre dicho tema, especialmente

(3) V. refs. 1 y 2.

(4) Pero dicha hipótesis dejan de tener validez: las secciones ya no se conservan normales a la directriz (aparte de otros efectos locales). 
para el caso de flexión pura, es decir, para la relación entre $\mathbf{M}$ y $\chi$ con $\mathbf{N}=0$ (leyes "momentos-curvatura"). No se expone aquí, pues, cómo estimar dichas leyes, interesándonos por el contrario en cómo pueden integrarse las mismas en el análisis del conjunto de la estructura.

A fin de tratar con leyes esfuerzos-deformaciones de la mayor generalidad, compatible con una razonable facilidad de procesamiento, se suponen dichas leyes idealizadas en forma de leyes multilineales ("bi-lineales", "tri-lineales", etc.), tal como se indica esquemáticamente en la figura I.1.1. Esta idealización es normalmente posible con pocos intervalos rectilíneos, ya que las secciones habituales suelen presentar fases de comportamiento bien definidas (estados "elástico", "fisurado" y «plástico»), con transiciones relativamente bruscas.

Destaquemos las principales características de las leyes esquematizadas en la figura 1.1.1.

a) Se relacionan por un lado el momento flector, M, y la curvatura, $\chi$; y por otro, el esfuerzo axil, $\mathbf{N}$, y la elongación unitaria, $\varepsilon$ (las dos deformaciones, $\varepsilon$ y $\chi$, se refieren a la directriz de las piezas; pero, en la hipótesis de deformación plana, ambos parámetros son suficientes para caracterizar la deformación global de las secciones).

b) Además, los diagramas momentos-curvatura se suponen dependientes paramétricamente del valor de N. Y los diagramas axil-elongación, del valor de $\mathbf{M}$. Naturalmente, el caso de curvas unívocas se obtiene como un caso particular (1). c) A cada pareja de deformaciones $\varepsilon$ y $\chi$ se asigna una pareja bien determinada de valores de los esfuerzos $\mathbf{N}$ y $\mathbf{M}$. No se considera, por lo tanto, la influencia de las deformaciones diferidas de los materiales, aunque las mismas (normalmente, tan sólo la fluencia del hormigón) pueden tenerse aproximadamente en cuenta (en su caso) ampliando la escala de abscisas en la ley $\sigma-\varepsilon$ del hormigón utilizada como base para el análisis previo de la sección.

d) Por la misma razón especificada en el párrafo anterior, los diagramas propuestos no son aptos para la consideración de fases de carga y descarga, sino sólo para la actuación de cargas estáticas crecientes hasta el agotamiento de la estructura. No obstante, sirven de base para el análisis de ésta en todas sus fases de comportamiento (no sólo para el análisis límite).

e) Las leyes $\mathbf{M}-\chi$ y $\mathbf{N}-\varepsilon$ se suponen estrictamente crecientes, de forma que las rigideces tangentes, $\Delta \mathbf{M} / \Delta \chi, \Delta \mathbf{N} / \Delta \varepsilon$, sean no nulas y no negativas. Sin embargo:

(1) Aunque las leyes $M-\chi$ dependan efectivamente de $\mathrm{N}$, es claro que para piezas con axil nulo o pequeño basta dar una curva $M-\chi$ única. En general, no hay necesidad de especificar el comportamiento de la sección más que para el rango de esfuerzos previsto para la estructura y acciones en cuestión: así, en zonas con flexiones «negativas" exclusivamente, es posible ahorrarse el cálculo de la rama de la curva $\mathrm{M}-\chi$ para $\mathrm{M}>0$ (y viceversa); o, si los axiles permanecen elásticos, no hay necesidad de definir las ramas plásticas de las curvas $\mathrm{N}-\varepsilon$, etc.
Fig. I.1.1

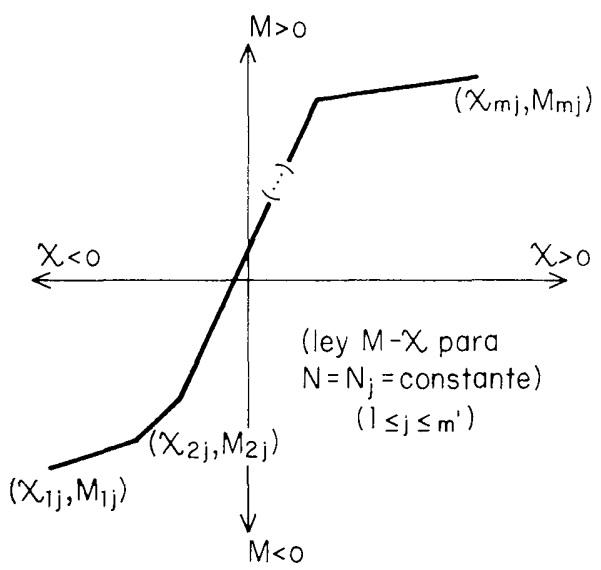

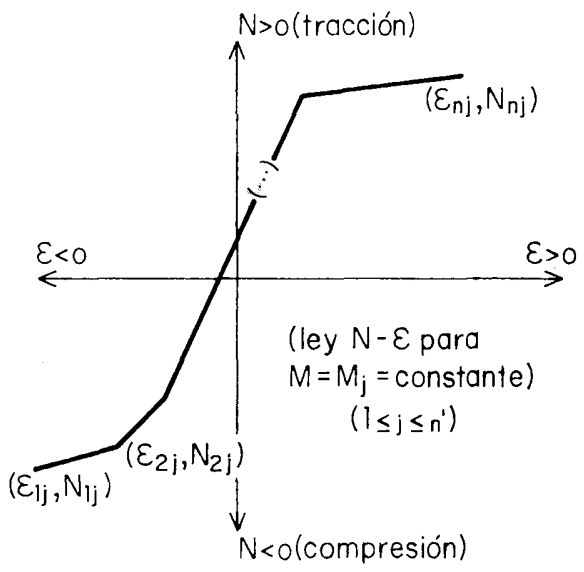


- el valor de dichas rigideces puede teóricamente hacerse tan pequeño como se desee (en la práctica esto tiene un límite, por exigencia de buen condicionamiento de los sistemas de ecuaciones a resolver) (1);

- dichas rigideces pueden, sin embargo, variar de cualquier forma, no tienen necesariamente por qué decrecer a medida que aumentan los esfuerzos.

f) No se exige que los diagramas $\mathbf{N}-\varepsilon$ y $\mathbf{M}-\chi$ "pasen por el origen". Es posible así tener adecuadamente en cuenta el efecto de predeformaciones impuestas a parte de los materiales, estando ya establecida la continuidad de la estructura (pretensado, gradientes térmicos, etc.) (2). Y también representar adecuadamente situaciones en las que, por ejemplo, se consideren varias curvas $\mathbf{M}-\chi$ para valores diferentes de $\mathbf{N}$, algunos de los cuales produzcan por sí solos (para $\mathbf{M}=0$ ) fisuraciones o plastificaciones: entonces, manteniendo constante la directriz de la pieza, no es posible en general (salvo para secciones simétricas) conseguir que todas las curvas $\mathbf{M}-\chi$ pasen por el origen (es decir, parte de ellas presentarán un valor $\mathbf{M} \neq 0$ para $\chi=0$ ).

g) Puesto que las leyes $\mathbf{M}-\chi$ y $\mathbf{N}-\varepsilon$ se suponen deducidas de acuerdo con la hipótesis de deformación plana, no se introducen en principio deformaciones debidas al esfuerzo cortante. La influencia de éste en los valores últimos de $\mathbf{N}$ y $\mathbf{M}$, puede introducirse limitando éstos por debajo de los valores calculados con cortante nulo (utilizando, por ejemplo, diagramas de interacción deducidos plásticamente), siempre que se prevea una influencia desfavorable de dicho esfuerzo.

Si las deformaciones por cortante son de consideración, puede incorporarse en el cálculo el correspondiente incremento de la flexibili-

(1) - Sin embargo, pueden resolverse perfectamente casos en los que dichas rigideces varíen como de 1 a $10^{5}$ o incluso más, sin ello exigir niveles especiales de precisión en las variables numéricas utilizadas por el ordenador.

(2) También es perfectamente posible considerar otros tipos de predeformaciones o presolicitaciones (por ejemplo, el hormigonado de una pieza mixta sin apeos del acero estructural) que alteran los diagramas esfuerzos-deformaciones, pero no su "paso por el origen". dad de la pieza, y en su caso, las correspondientes deformaciones de tipo plástico.

La técnica de cálculo es esencialmente iterativa: partiendo de los esfuerzos de la iteración precedente (inicialmente, de los esfuerzos "elásticos»), se establece una partición de cada barra en zonas en las que el estado de las secciones pueda considerarse constante (aplicación de una rama única de las leyes esfuerzos-deformaciones); analizados por separado cada uno de estos elementos de barra, sus "respuestas" individuales se combinan según una técnica análoga a la de las matrices transferencia (3). Aplicando unas transformaciones sencillas a las ecuaciones resultantes, las mismas se expresan en la forma:

$$
\begin{aligned}
& \mathbf{P}_{1}=\mathbf{K}_{11} \cdot \mathbf{d}_{1}+\mathbf{K}_{12} \cdot \mathbf{d}_{2}+\mathbf{q}_{1} \\
& \mathbf{P}_{2}=\mathbf{K}_{21} \cdot \mathbf{d}_{1}+\mathbf{K}_{22} \cdot \mathbf{d}_{2}+\mathbf{q}_{2}
\end{aligned}
$$

siendo $\mathbf{P}_{1}$ y $\mathbf{P}_{2}$ los vectores-esfuerzos de extremo de barra (v. ref. núm. 1), y $\mathbf{d}_{1}, \mathbf{d}_{2}$, los vectores-movimientos de extremo. Dichas ecuaciones son análogas a las del cálculo matricial elástico, pero:

- las matrices rigidez de barra $\left(\mathbf{K}_{11}, \mathbf{K}_{12}\right.$, $K_{21}, K_{22}$ ) se calculan en función de los esfuerzos en la iteración precedente, y son por lo tanto variables de una iteración a otra.

- aparecen los términos $\mathbf{q}_{1}, \mathbf{q}_{2}$ (vectoriales) si la barra presenta deformaciones de tipo plástico.

Basándose en las anteriores ecuaciones de respuesta de cada barra, es sencillo modificar los algoritmos del método de la rigidez en lo relativo a las ecuaciones generales de la estructura, dentro de la Teoría de primer orden. La consideración de los fenómenos de segundo orden exige más modificaciones, pero también es factible la adaptación correspondiente del algoritmo lineal.

\section{EJEMPLOS DE CALCULO}

En primer lugar se analiza el pórtico indicado en la figura 1.2.1, dentro de la Teoría de primer orden y con definición sencilla de las leyes momentos-curvatura: diagramas bilineales unívocos ( $\sin$ dependencia de $\mathbf{N}$ ), salvo

(3) V. ref. 1. 


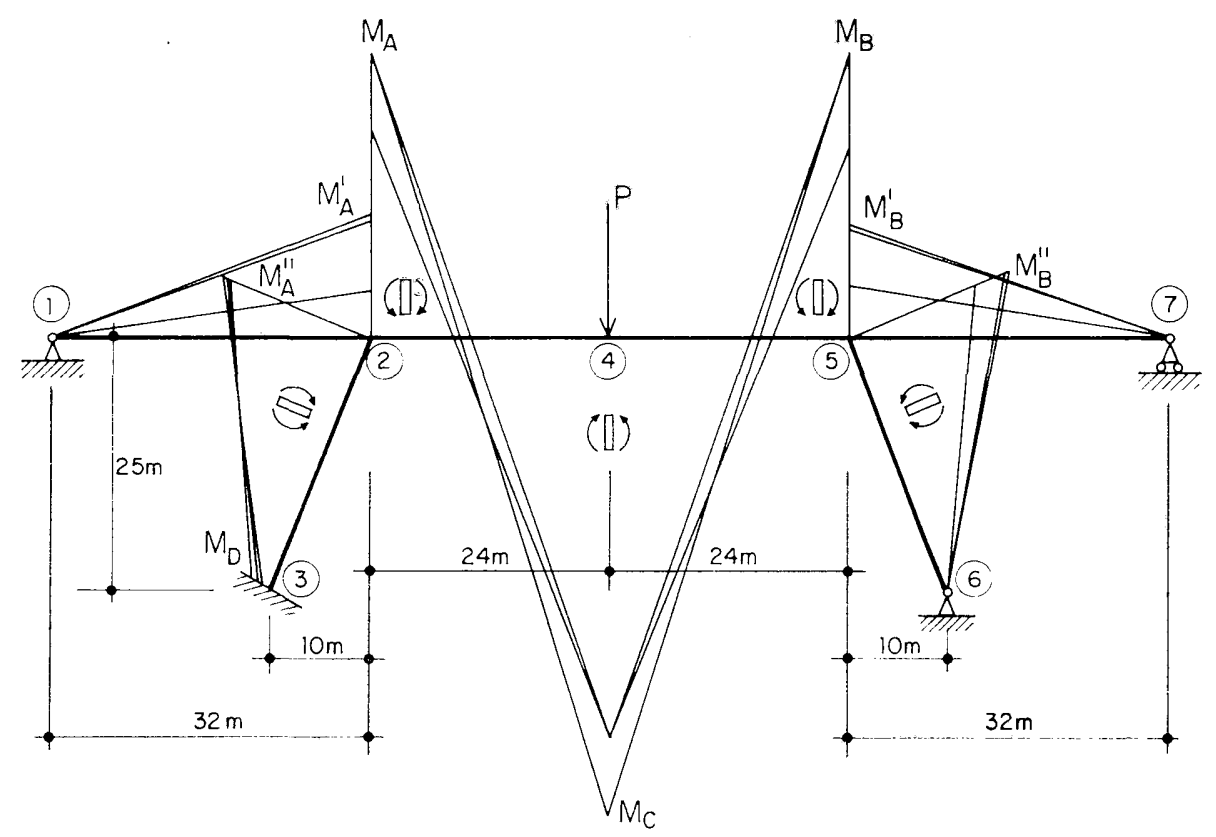

Fig. I.2.1

para las barras 2-3 y 5-6, para las cuales los valores últimos del momento y de la curvatura se ajustan según el axil, dentro de un intervalo de valores estimado para cubrir los axiles resultantes con los valores supuestos de la carga P. Los cálculos se han efectuado con las leyes momentos-curvatura definidas por la tabla siguiente:

\begin{tabular}{|c|c|c|c|c|c|c|c|c|c|c|c|}
\hline \multicolumn{5}{|c|}{$\mathbf{m} \cdot \mathbf{M p}$} & \multicolumn{5}{|c|}{$\mathbf{m}^{-1}$} & \multirow{2}{*}{$\begin{array}{c}\text { Para } \\
\text { un axil } \mathbf{N} \\
(\mathrm{Mp})\end{array}$} & \multirow{2}{*}{ Barras } \\
\hline$M_{1}$ & $\mathbf{M}_{2}$ & $M_{3}$ & $M_{4}$ & $M_{5}$ & $\chi_{1}$ & $\gamma_{2}$ & $x_{3}$ & $\chi_{4}$ & $\chi_{5}$ & & \\
\hline-225 & -195 & 0 & 195 & 225 & $-0,02$ & $-0,00005$ & 0 & 0,000025 & 0,01 & - & $1-2$ y $5-7$ \\
\hline-425 & -370 & 0 & 725 & 800 & $-0,016$ & $-0,00005$ & 0 & 0,000055 & 0,008 & - & $2-4$ y $4-5$ \\
\hline-275 & -235 & 0 & 235 & 275 & $-0,01$ & $-0,000025$ & 0 & 0,000025 & 0,01 & -75 & \\
\hline-270 & -230 & 0 & 230 & 270 & $-0,011$ & $-0,000026$ & 0 & 0,000026 & 0,011 & -50 & $2-3$ y $5-6$ \\
\hline-265 & -225 & 0 & 225 & 265 & $-0,012$ & $-0,000027$ & 0 & 0,000027 & 0,012 & -25 & \\
\hline
\end{tabular}

En esta tabla, los valores $\left(\chi_{j}, \mathbf{M}_{j}\right)$ son los correspondientes a los vértices del diagrama momentos-curvatura.

Los axiles se suponen en régimen elástico, si bien con valores diferentes de la rigidez “EA» $(=\mathbf{N} / \varepsilon)$ según el signo del esfuerzo (1):

(1) En realidad en este caso es fácil prever el signo del axil en todas las barras, con lo cual esa diferenciación es innecesaria.
Las características indicadas de las secciones no corresponden a ningún caso real, pero han sido elegidas dentro de márgenes potencialmente reales; con lo cual, el ejemplo pre-

\begin{tabular}{c|c|c}
\hline \multicolumn{2}{c|}{ “EA» $(\mathrm{Mp})$} & \multicolumn{1}{c}{ Barras } \\
\hline Compresión & Tracción & \\
\hline 4.666 .666 & 950.000 & $1-2$ y $5-7$ \\
\hline 7.000 .000 & 950.000 & $2-4$ y $4-5$ \\
\hline 7.000 .000 & 937.500 & $2-3$ y $5-6$ \\
\hline
\end{tabular}



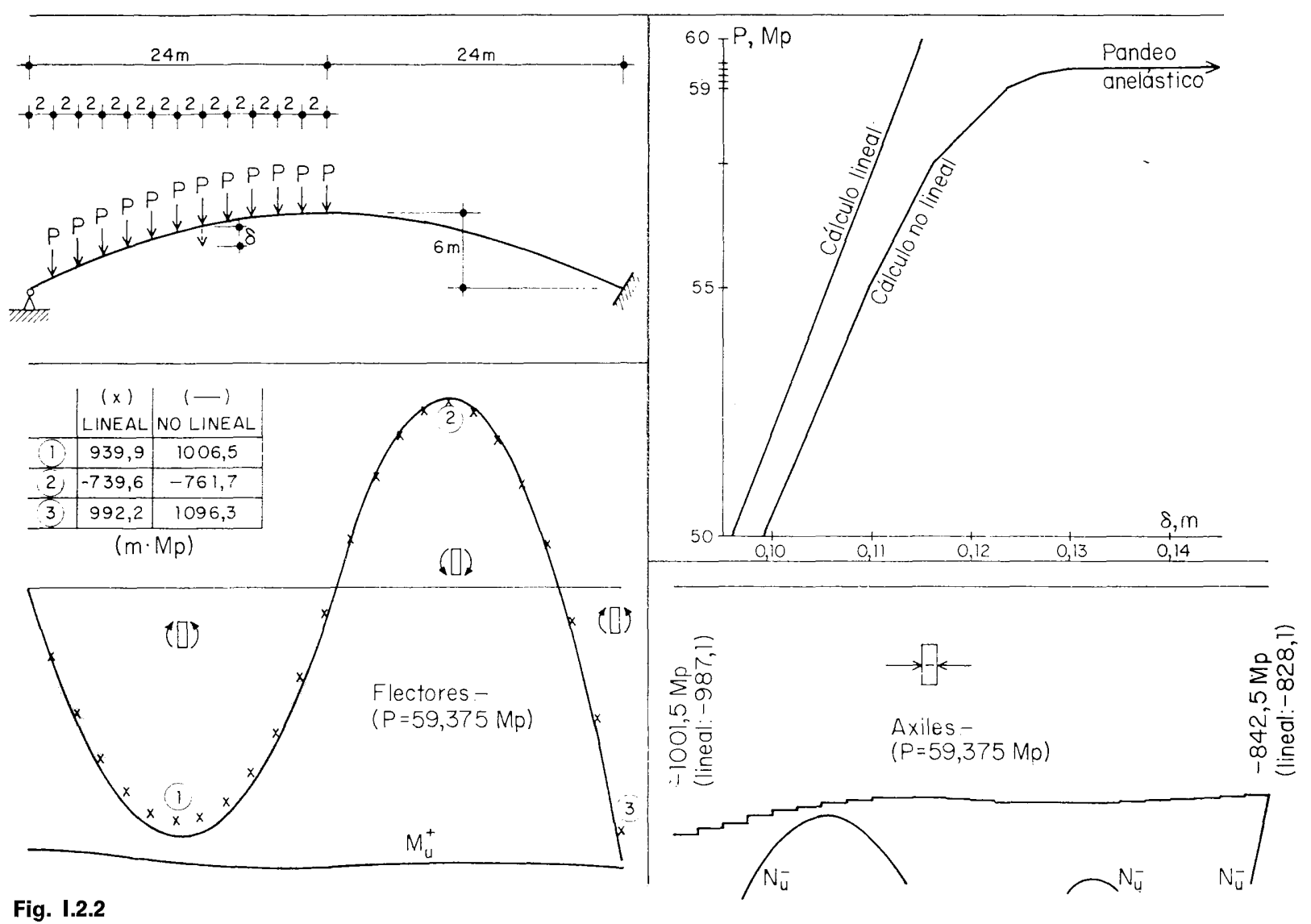

Fig. 1.2 .2

leyes "simétricas" (1) (es decir, suponiendo simétrica la sección) y bilineales. De esta forma, para definir dichas leyes basta con dar el "vértice" y el "extremo" de las mismas:

\section{LEY MOMENTOS-CURVATURA}

$\left(\mathrm{m} \cdot \mathrm{Mp}, \mathrm{m}^{-1}\right)$

\begin{tabular}{c|c|c|c|c}
\hline \multicolumn{2}{c|}{ Vértice } & \multicolumn{2}{c|}{ Valores últimos } & $\begin{array}{c}\text { Valor } \\
\text { del axil } \\
\text { Mp }\end{array}$ \\
\cline { 1 - 5 }$|\mathbf{M}|$ & $|\chi|$ & $\left|\mathbf{M}_{\mathrm{u}}\right|$ & $\left|\chi_{\mathrm{u}}\right|$ & \multicolumn{1}{c}{${ }^{2}$} \\
\hline 1225 & 0,00174 & 1400 & 0,02 & 0 \\
\hline 1137 & 0,00162 & 1300 & 0,02 & -500 \\
\hline 1028 & 0,00146 & 1175 & 0,01538 & -750 \\
\hline 919 & 0,00131 & 1050 & 0,01356 & -1000 \\
\hline 612 & 0,000869 & 700 & 0,01149 & -1250 \\
\hline 394 & 0,000559 & 450 & 0,01023 & -1500 \\
\hline 153 & 0,000217 & 175 & 0,01 & -1750 \\
\hline 43 & 0,000061 & 50 & 0,01 & -1900 \\
\hline
\end{tabular}

(1) Es decir, con $\mathbf{M}$ definido como función «impar" de $\chi$ (a $\mathbf{N}$ cte.); y $\mathbf{N}$, como función "par» de $\chi$ (a $\mathbf{M}$ cte.). 
sentado tiene interés no solamente como prueba del algoritmo de cálculo, sino también como muestra de la adaptación anelástica de las estructuras reales de grandes dimensio- nes. En la propia figura I.2.1 se representan gráficamente las leyes de flectores obtenidas para tres niveles de carga; numéricamente, dichos resultados son:

\begin{tabular}{c|c|c|c|c|c|c}
\hline $\mathbf{m} \cdot \mathbf{M p}$ & $\mathbf{P}=\mathbf{7 5} \mathbf{M p}$ & $\mathbf{P}=\mathbf{8 5} \mathbf{M p}$ & $\mathbf{P}=\mathbf{9 5} \mathbf{M p}$ & \multicolumn{3}{|c}{ (Valores «elásticos") } \\
\hline & & & & $\mathbf{P}=\mathbf{7 5} \mathbf{M p}$ & $\mathbf{P}=\mathbf{8 5} \mathbf{M p}$ & $\mathbf{P}=\mathbf{9 5} \mathbf{M p}$ \\
\hline $\mathrm{M}_{\mathrm{A}}$ & -310 & $-420,3$ & $-426,5$ & $-356,6$ & $-404,1$ & $-451,7$ \\
\hline $\mathrm{M}_{\mathrm{A}}^{\prime}$ & $-70,2$ & $-177,7$ & $-182,6$ & $-132,3$ & -150 & $-167,6$ \\
\hline $\mathrm{M}^{\prime \prime}{ }_{\mathrm{A}}$ & $-239,8$ & $-242,6$ & $-243,9$ & $-224,3$ & $-254,1$ & $-284,1$ \\
\hline $\mathrm{M}_{\mathrm{B}}$ & $-284,2$ & $-420,4$ & $-426,5$ & $-327,6$ & $-371,2$ & $-414,9$ \\
\hline $\mathrm{M}^{\prime}{ }_{\mathrm{B}}$ & $-77,6$ & $-164,6$ & $-169,5$ & $-144,3$ & $-163,5$ & $-182,7$ \\
\hline $\mathrm{M}^{\prime \prime}{ }_{B}$ & $-206,6$ & $-255,8$ & -257 & $-183,3$ & $-207,7$ & $-232,2$ \\
\hline $\mathrm{M}_{\mathrm{C}}$ & 602,9 & 599,6 & 713,5 & 557,9 & 632,3 & 706,7 \\
\hline $\mathrm{M}_{\mathrm{D}}$ & $-24,7$ & $-13,9$ & $-12,9$ & 107,8 & 122,2 & 136,6 \\
\hline
\end{tabular}

Los valores "elásticos" indicados en la tabla anterior son los que se obtienen operando con las siguientes rigideces de las secciones:

- a flexión, la rigidez de la rama $\mathbf{M}-\chi$ para flectores positivos en fase "elástica" (para $\mathbf{N}=0$, o para el valor de $\mathbf{N}$ más próximo a cero, en caso de diagramas variables con el axil);

- frente a deformaciones axiles, la rigidez de la rama $\mathbf{N}-\varepsilon$ para compresiones en fase "elástica».

Observando los resultados anteriores se deduce que, para la estructura en cuestión, las sucesivas redistribuciones de esfuerzos se producen debido a:

- la reducción de rigidez por flexión negativa en las barras 1-2, 5-7, 2-3, 5-6 (y, parcialmente, las 2-4 y $4-5$ );

- la entrada en fase plástica del extremo "2" de la barra 2-3;

- ídem del extremo "2" de la barra 2-4, del “5" de la 4-5 y del extremo «5" de la 5-6.

En cambio, no llega a formarse la rótula plástica "positiva" en el nudo "4". En efecto, cuando $\mathbf{M}_{\mathrm{C}}$ vale un $89,2 \%$ de su valor de rotura, $\mathbf{M}_{\mathrm{A}}$ у $\mathbf{M}_{\mathrm{B}}$ han alcanzado ya el $100 \%$ de su valor último. De esta forma, el valor de agotamiento de la carga $\mathbf{P}$ es aproximadamente de $95 \mathrm{Mp}$, es decir, un $93 \%$ de la carga prevista por la Teoría de la Plasticidad $(\mathbf{P}=102,1 \mathrm{Mp})$ y un $106,4 \%$ de la calculable con los esfuerzos elásticos $(\mathbf{P}=89,3 \mathrm{Mp}$, que da un $\mathbf{M}_{\mathrm{A}}^{\prime}$ "elástico" igual a $425 \mathrm{~m} \cdot \mathrm{Mp}$ ). Los movimientos calculados se incrementan notablemente respecto a los "elásticos" (por ejemplo, 3,16 veces para el descenso del nudo " 4 ").

En cuanto a la convergencia del proceso de cálculo, con tan sólo cuatro iteraciones se obtienen errores del orden del $0,1 \%$ para los esfuerzos y movimientos máximos en valor absoluto (y ligeramente superiores para los de menor importancia).

Como segundo ejemplo de cálculo se presenta el caso del arco articulado-empotrado de directriz parabólica indicado en la figura I.2.2, con las cargas especificadas en esta misma figura. Se ha considerado al efecto una única sección tipo, con una familia completa de diagramas momentos-curvatura para axil de compresión, y de diagramas axil-elongación para todo el rango de flectores; y adoptando 
de la $\mathbf{N}_{\mathrm{u}}{ }^{-}$a las leyes de flectores y axiles corresponde a las secciones más cercanas al agotamiento.

\section{ANALISIS NO LINEAL DE LAMINAS DE REVOLUCION DE HORMIGON ARMADO}

De los diferentes métodos estudiados en la referencia 13 para el análisis no lineal de las láminas de revolución de hormigón armado, el más interesante (a pesar de requerir medios potentes de cálculo automático) es un método "matricial" de gran similitud formal con el algoritmo antes comentado para el análisis no lineal de estructuras de barras. Sus ventajas radican en su exactitud y versatilidad en lo referente a geometrías posibles, variedad de condiciones de sustentación y representación precisa de los diferentes estados anelásticos posibles en el interior de la lámina; además, su planteamiento no exige un aparato conceptual sustancialmente distinto al que es habitual en el cálculo de estructuras de barras.

En la figura II.1.1 pueden verse los esfuerzos actuantes sobre un elemento de lámina comprendido entre dos meridianos y dos paralelos a distancia infinitesimal (1), esfuerzos que son en esencia los mismos que habitualmente se consideran sobre una pieza de una estructura de barras (sin más que medirlos por unidad de longitud del paralelo respectivo), más unos axiles $\left(\mathbf{N}_{\theta}\right)$ y flectores $\left(\mathbf{M}_{\theta}\right)$ actuantes en la dirección ortogonal y medidos por unidad de longitud de meridiano. El resto de los posibles esfuerzos actuantes sobre dicho elemento de lámina son nulos, en vir-

(1) Para el axil, cortante y flector según los meri dianos $\left(\mathbf{N}_{\infty}, \mathbf{Q}_{\infty}, \mathbf{M}_{\infty}\right)$ se observará que se adopta un criterio de signos distinto en los dos extremos del elemento, convenio que es habitual en el cálculo matricial de estructuras. tud de la simetría del problema (las acciones y sustentación se supone que respetan dicha simetría).

Las hipótesis básicas del método son las siguientes:

- Se supone que el meridiano tiene forma poligonal, siendo recta la directriz de cada elemento de lámina (fig. II.1.1). Esta hipótesis no es restrictiva en la práctica, siempre que se cuente con ordenadores lo suficientemente potentes como para permitir una discretización del meridiano de la lámina tan fina como sea preciso (2).

- Se supone asimismo que la longitud de cada elemento de meridiano es lo bastante pequeña como para que puedan aceptarse variaciones lineales de los esfuerzos entre los dos paralelos extremos de cada elemento (2).

- Cargas actuantes exclusivamente en los paralelos de separación entre elementos. En caso de cargas repartidas, su discretización no afecta prácticamente a los resultados, siempre que dichos paralelos estén suficientemente próximos (2).

- Relaciones esfuerzos-deformaciones de tipo trilineal, con parámetros dependientes del esfuerzo axil. Nulidad del efecto Poisson.

La simplificación de prescindir del efecto Poisson apenas afecta al análisis de una lámina de revolución, al menos en caso de que el material de la misma sea el hormigón armado; el valor real del coeficiente de Poisson puede, no obstante, tenerse en cuenta para

(2) En las pruebas de cálculo efectuadas, con longitudes del elemento de meridiano $(\Delta \mathbf{s})$ del orden de dos a tres veces el canto de la lámina, los errores observados han sido insignificantes.

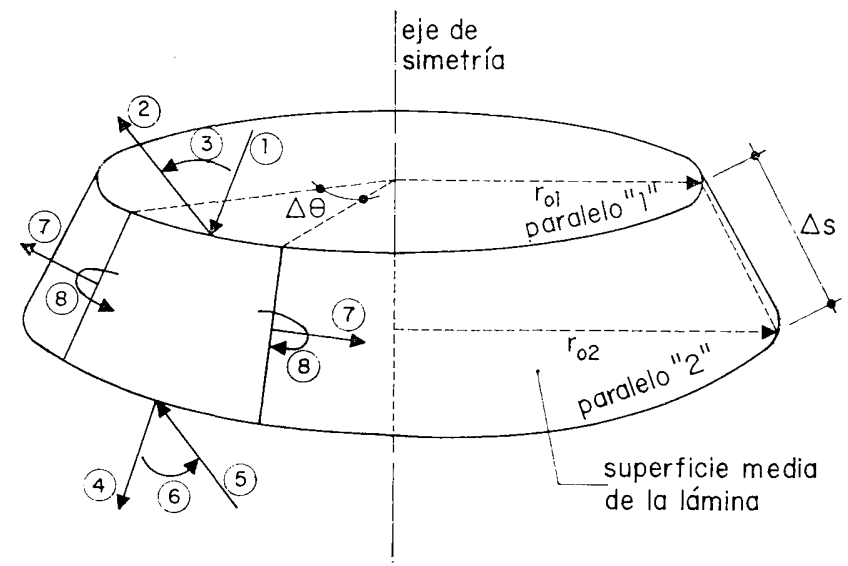

$$
\begin{aligned}
& \text { (1) }=P_{1 x} r_{01} \Delta \theta \\
& \text { (2) }=P_{1 y} r_{01} \Delta \theta \\
& \text { (3) }=P_{1 z} r_{01} \Delta \theta \\
& \text { (4) }=P_{2 x} r_{02} \Delta \theta \\
& \text { (5) }=P_{2 y} r_{02} \Delta \theta \\
& \text { (6) }=P_{2 z} r_{02} \Delta \theta \\
& \text { (7) }=N_{\theta} \Delta s \\
& \text { (8) }=M_{\theta} \Delta s
\end{aligned}
$$

Fig. II.1.1 
la evaluación "a posteriori» de algún esfuerzo secundario $\left(\mathbf{M}_{\theta}\right)$.

La disposición de armaduras junto a ambos paramentos de la lámina se supone simétrica respecto a la superficie media de ésta; y la orientación de las armaduras, coincidente con la de meridianos y paralelos.

La forma, esquemática, de las leyes trilineales propuestas (ajustables de forma continua en función del esfuerzo axil), puede verse en la figura II.1.2.

El estudio de la "respuesta" anelástica de cada elemento de lámina es más complejo que el de una barra prismática; pero las ecuaciones resultantes son también de la forma

$$
\begin{aligned}
& \mathbf{P}_{1}=\mathbf{K}_{11} \cdot \mathbf{d}_{1}+\mathbf{K}_{12} \cdot \mathbf{d}_{2}+\mathbf{q}_{1}, \\
& \mathbf{P}_{2}=\mathbf{K}_{21} \cdot \mathbf{d}_{1}+\mathbf{K}_{22} \cdot \mathbf{d}_{2}+\mathbf{q}_{2},
\end{aligned}
$$

a partir de las cuales las ecuaciones generales de la lámina se forman mediante una técnica similar a la del método de la rigidez de las estructuras de barras; también es adaptable el método de las matrices transferencia.

El cálculo se efectúa de forma iterativa, ya que la "respuesta" de cada elemento de lá- mina se establece en función de los esfuerzos calculados en la iteración precedente (inicialmente, a partir de los esfuerzos "elásti$\cos ")$.

\section{EJEMPLO DE CALCULO}

En la figura II.2.2 se indican las leyes de esfuerzos obtenidos para la lámina en casquete esférico cuya sección meridiana se representa en la figura II.2.1. Las coacciones en los bordes se han elegido del tipo de empotramientos deslizantes (en un plano horizontal el exterior; y en un plano vertical el interior) a fin de producir un importante efecto de borde y poner así claramente de manifiesto los fenómenos anelásticos. El análisis se ha efectuado según la Teoría de primer orden.

El canto es constante e igual a $10 \mathrm{~cm}$. El hormigón se ha supuesto con una resistencia en compresión de $240 \mathrm{kp} / \mathrm{cm}^{2}$ y un módulo de elasticidad (fase "l") de $3 \times 10^{5} \mathrm{kp} / \mathrm{cm}^{2}$. Las armaduras, con un límite elástico de $4.400 \mathrm{kp} / \mathrm{cm}^{2}$ y un módulo de elasticidad de $2,1 \times 10^{6} \mathrm{kp} / \mathrm{cm}^{2}$; la distribución de las mismas se supone simétrica respecto a la superficie media, con una cuantía de $\boldsymbol{A}_{\phi} \mathrm{cm}^{2} / \mathrm{m} . \mathrm{I}$. junto a cada paramento, y una distancia de

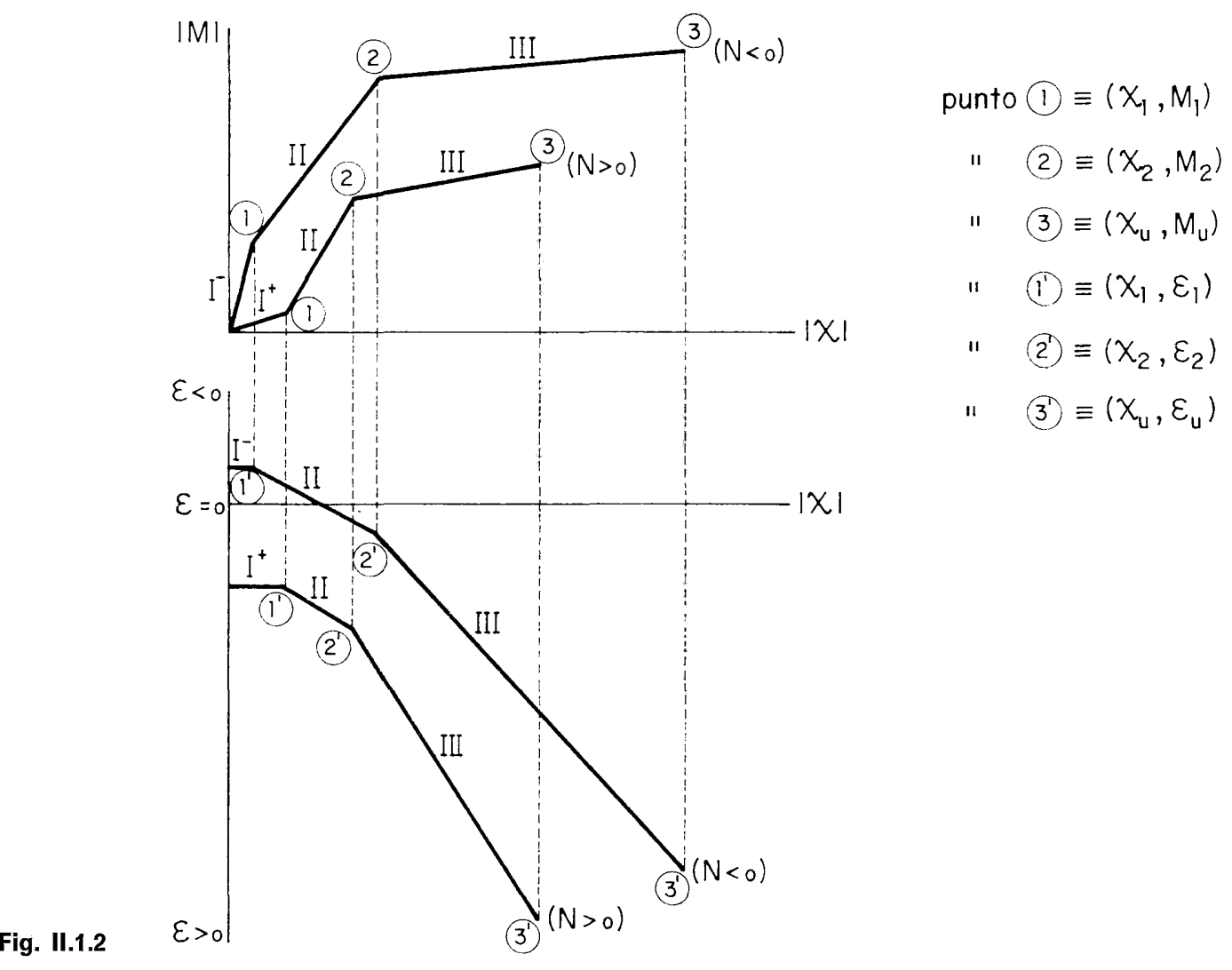


$\mathbf{h}_{\phi} \mathrm{cm}$ entre los c.d.g. de los dos niveles de armaduras:

- Según los meridianos, para

$$
0<\mathbf{X}<5,25 \mathrm{~m} ; \mathbf{A}_{\phi}=5,96 \mathrm{~cm}^{2} / \mathrm{m} \text {, }
$$$$
\mathbf{h}_{\phi}=7 \mathrm{~cm} \mathrm{(1)} \text {. }
$$

- Según los meridianos, para

$$
5,25<\mathbf{X}<9 \mathrm{~m} ; \mathbf{A}_{\phi}=3,27 \mathrm{~cm}^{2} / \mathrm{m} \text {, }
$$$$
\mathbf{h}_{\phi}=7 \mathrm{~cm} \text {. }
$$

- Según los paralelos, en toda la lámina:

$$
\mathbf{A}_{\phi}=3,27 \mathrm{~cm}^{2} / \mathrm{m}, \mathbf{h}_{\phi}=5 \mathrm{~cm} \text {. }
$$

Es fácil comprobar que esta distribución de armaduras no está proporcionada según un dimensionamiento en base a leyes "elásticas" de esfuerzos (lo cual se ha dispuesto intencionadamente para acentuar aún más los efectos anelásticos).

Las leyes de esfuerzos indicados corresponden a $\mathbf{q}=0,25 \mathrm{Mp} / \mathrm{m}^{2}$.

Las leyes "elásticas" son las obtenidas con el mismo algoritmo "matricial", pero suponiendo un comportamiento lineal de las secciones, con rigideces correspondientes al estado $1^{-}$del material (sin fisuración). Por ser sencilla la geometría de la lámina, existen soluciones analíticas aproximadas del problema elástico (Geckeler) (2), que concuerdan con las leyes "elásticas" aquí indicadas (estas leyes constituyen precisamente la primera iteración del cálculo no lineal de la lámina).

Las causas de las redistribuciones de los esfuerzos observables en la figura II.2.2 son la existencia de una amplia zona con $\mathbf{N}_{\theta}>0$ (fisuración) y la entrada en fase "ll" de los flectores $\mathbf{M}_{r}$. La ley de axiles $\mathbf{N}_{\theta}$ presenta una zona de tracciones notablemente superior a la de las leyes elásticas, y una importante reducción de $\mathbf{N}_{\theta}$ en el borde exterior; a partir del punto en que dicho esfuerzo entra en compresión, la curva correpondiente experimenta un quiebro con incremento de la pendiente y reducción de la "longitud de onda" (es decir, sufre una auténtica "refracción"); la variabilidad de los restantes esfuerzos en la misma zona se ve también afectada, aunque en menor grado. La forma de las leyes de axiles $\mathbf{N}_{\psi}$ y de cortantes es similar a la de las leyes

(1) $\mathbf{x}=$ distancia (en planta) al borde exterior (ver figura II.2.1)

(2) Ver ref. núm. 7 .

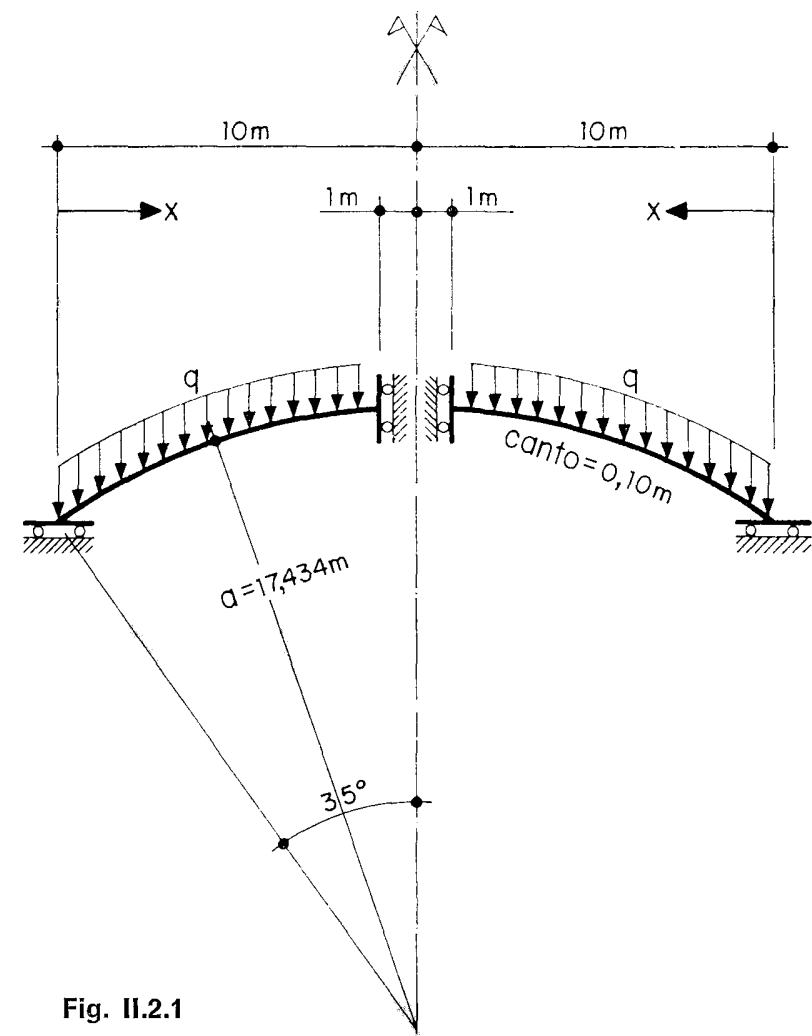

elásticas, si bien con mayor "longitud de onda"; los valores en los bordes coinciden con los elásticos, debido a la particular sustentación de la lámina analizada. La forma de la ley de flectores $\mathbf{M}_{\varphi}$ es también similar a la de la ley elástica, con amplificación de la "longitud de onda"; y experimenta un importante incremento en el borde (el trabajo tipo arco se acentúa al hacerse menos rígidos los paralelos].

Incrementando el valor de q se llega a produ$\operatorname{cir}$ (para $\mathbf{q} \simeq 0,7 \mathrm{Mp} / \mathrm{m}^{2}$ ) una zona plastificada de la armadura según los paralelos $\left(\mathbf{N}_{\theta}=\mathbf{N}_{\theta \mathrm{u}}\right.$ ), con lo cual el reajuste de $\mathbf{N}_{\theta}$ respecto a su valor elástico es todavía más acusado. También $\mathbf{M}_{\varphi}$ entra en el borde exterior en fase plástica; de hecho, para el nivel de carga indicado $\left(\mathbf{q} \simeq 0,7 \mathrm{Mp} / \mathrm{m}^{2}\right)$, la deformabilidad de la rótula plástica correspondiente puede considerarse agotada.

\section{REFERENCIAS}

1. "Métodos matriciales para el cálculo de estructuras". R. K. Livesley (Blume, 1970).

2. "Análisis de estructuras reticulares". J. M. Gere, W. Weaver (C. Editorial Continental, 1967). 


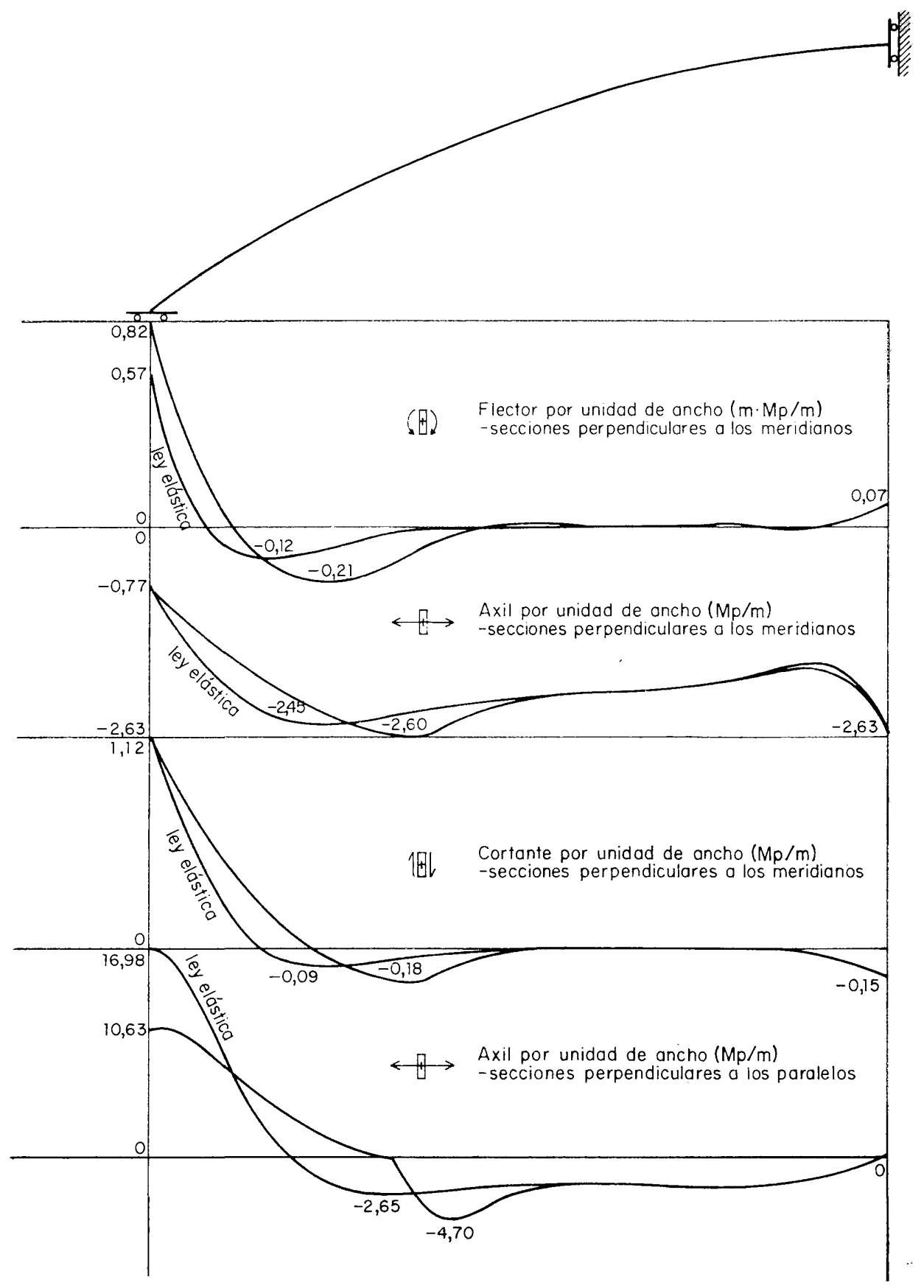

Fig. 11.2 .2

3. "Cálculo plástico de las construcciones". Ch. Massonnet, M. Save (Montaner y Simın, 1966).

4. "Elasticity and Plasticity". Naismith, Stanley, Freudenthal, Geiringer, Reiner. Encyclopedia of Physics, vol. VI (Springer Verlag, 1958).

5. "Theoretical Elasticity". A. E. Green, W. Zerna (Oxford, 1968).

6. "Mathematical Theory of Elasticity". I. S. Sokolnikoff (McGraw Hill, 1956).

7. “Teoría de placas y láminas». S. Timoshenko, S. Woinowsky-Krieger (Urmo, 1970).

8. "Comportamiento y cálculo anelástico de las estructuras hiperestáticas de hormigón armado y pretensado». J. Martínez Calzón (Monografías del I.E.T.c.c., núm. 302, 1972).
9. "Manuel de Calcul "Fissuration" ". C.E.B. Boletín de Información núm. 89, 1973.

10. "Programación lineal». M. Simonnard (Paraninfo, 1972).

11. "Elements of Numerical Analysis». J. Singer (Academic Press, 1968).

12. "Elementary Numerical Analysis». S. D. Conte, C. de Boor (McGraw Hill K., 1972)

13. "Estudio del efecto de la no linealidad esfuerzosdeformaciones en la distribución de solicitaciones y movimientos en estructuras laminares de hormigón armado con simetría de revolución de forma, características, sustentación y acciones". J. Ortiz H. (Tesis doctoral en la E.T.S. de I.C.C.P. de Madrid). 


\section{résumé}

Calcul non linéaire des structures réticulaires et des voilles minces

Jesús Ortiz Herrera, Dr. ingénieur des Ponts et Chaussées

A l'aide du calcul électronique, I'analyse non linéaire des structures de barres est A dans les limitations du calcul plastique tra ditionnel Afin de prouver cotte affirmation, ditiósente de prouver cette affirmation, effectuées suivant des techniques et des programmes de calcul propres. On présente également l'analyse non linéaire des voiles à révolution auxquels sont applicables algorithmes de grande similitude formelle avec ceux du cas des structures de barres. ant ceur du cas dères structures que pour les voiles minces, les programmes développés par l'auteur permettent d'effectuer un grande diversité d'analyses anélastiques (et élastiques comme ras particulier).

\section{summary}

Non linear calculus of framed and laminated structures

Jesús Ortiz Herrera, Dr. Civ. Eng

By menas of electronic calculus it is possible to approach the non linear analysis of bar constructions efficiently and without the limitations of the traditional plastic Calculus. In order to prove this statement, the author presents certains structural analysis that have been carried out by means of tech niques and calculation programs of his own. Further, the non linear analysis of his own. sheets is presented to which algorithms of great formal similarity to those in the of bar constructions are applicable. cas for these latter ones as well as for laminated structures the programs elaborated by the author allow a great variety of anelastic analyses (and as a special case, of elastic analysis\}.

\section{zusammenfassung}

Nicht lineare Berechnung von Stockwerkrahmen und Flächentragwerken Jesús Ortiz Herrera, Dr. Hoch Tiefb. Ing.

Mittelst elektronischer Berechnung ist es möglich, die nicht lineare Analyse von Stab. konstrule Begrenzungen der traditionellen plastischen Berechnungen, zu verwenden Um diese Be gewisse Strukturanalysen, die hauptung zu prüfen, präsentiert der Autor eigener technischen Methoden und Berechnungsprogramme ausgeführt hat Ebenalls wird die nicht lineare Analyse von Dreh. folien prësentiert, are Analyse von Drehvon grosser formeller Ähnlichkeit mit denen des Stabkonstruktionfalles anwendbar sind. Für diese letzteren, wie für die Flächentrakwerke von dem Autor erarbeiteten Programme, gibt es eine grosse Variation von gramme, gibt es eine grosse Variation von Sonderfall].

\section{publicación del i.e. t. c.c.}

A. M. Haas

Dr. Ingeniero

Traducción de José M.a Urcelay Dr. Ingeniero de Caminos, Canales y Puertos

El profesor A. M. Haas es personalidad muy conocida en todo el mundo dentro del campo de las estructuras laminares.

El libro, que ha sido traducido a varios idiomas, es de exposición clara e intuitiva, y destaca los conceptos fundamentales sobre los desarrollos matemáticos.

En su primera parte, el libro trata de la teoría de membrana en láminas de revolución. A continuación se aplica esta teoría, para el caso en que las cargas sean también de revolución, a las láminas de revolución más usuales: cúpulas esférica y elíptica, láminas cónicas, depósitos.

Se estudian seguidamente las láminas de revolución sometidas a cargas que no sean de revolución, así como las tensiones secundarias debidas a flexiones en láminas de revolución.

Se termina la primera parte con un capítulo dedicado a la construcción de láminas.

En la segunda parte se estudia la teoría de membrana para láminas rebajadas, dedicando sendos capítulos a las láminas en paraboloide hiperbólico, en paraboloide elíptico y en conoide.

A continuación se dedica un extenso capítulo a la flexión.

Seguidamente se estudia el caso de pequeñas cargas que originan fuertes tensiones por flexión.

Finalmente, el libro dedica un capítulo al pandeo.

Un volumen encuadernado en tela, brillantemente presentado, de $17 \times 24,5 \mathrm{~cm}$, compuesto de 420 páginas, numerosas figuras, tablas y ábacos. Precios: España, 1.250 ptas.; extranjero, \$ 25. 